\title{
Dielectric relaxations of Acrilyc-Polyurethane hybrid materials
}

\author{
G. Martínez-Rugerio ${ }^{\mathrm{a}}$, Ángel Alegría ${ }^{\mathrm{a}, \mathrm{b}}$, Vesna Daniloskac ${ }^{\mathrm{c}}$, Radmila \\ Tomovska $^{\mathrm{c}, \mathrm{d}}$, M. Paulis ${ }^{\mathrm{c}}$, Juan Colmenero ${ }^{\mathrm{a}, \mathrm{b}}$ \\ ${ }^{a}$ Centro de Física de Materiales (CSIC-UPV/EHU)-Materials Physics Centre (MPC), \\ Paseo Manuel Lardizábal 5, 20018, San Sebastián, Spain. \\ ${ }^{b}$ Departamento de Física de Materiales, Universidad del País Vasco (UPV/EHU) \\ Apartado 1072, 20080, San Sebastián, Spain. \\ ${ }^{c}$ Polymat and Departamento de Química Aplicada, Facultad de Ciencias Químicas, \\ University of the Basque Country UPV/EHU, Jose Mari Korta Zentroa, Tolosa \\ Hiribidea 72, Donostia / San Sebastian, Spain. \\ ${ }^{d}$ IKERBASQUE, Basque Foundation for Science, 48011 Bilbao, Spain. \\ ${ }^{e}$ Donostia International Physics Centre, Paseo Manuel Lardizábal 4, 20018, San \\ Sebastian, Spain.
}

\begin{abstract}
In this work we present a dielectric relaxation study of Acrilyc/Polyurethane polymers synthesized via miniemulsion photo-polymerization. Three different samples, both parts of the synthesized hybrid latex (SOL and GEL fractions) and a full acrylic sample as reference have been considered. Besides dielectric experiments using broadband Dielectric Spectroscopy (BDS) technique, complementary Differential Scanning Calorimetry measurements have been performed. The thermodynamic characterization outcomes evidence very similar characteristics of the glass transition phenomena among the investigated samples without any signature of melting/crystallization. However BDS experiments show three well resolved dielectric relaxation processes above $\mathrm{Tg}$, two of them evidence marked differences among the samples. A detail study allows us to provide a molecular origin for these three dielectric relaxation processes in connection with the sample characteristics. In particular, our results show that the characterization of these hybrid Acrylic/PU by BDS can provide access to some of the structural features that would ultimately influence the adhesives properties.
\end{abstract}

Keywords: Dielectric Relaxation, Polymer dynamics, Acrylic/Polyurethane hybrids. 


\section{Introduction}

Self-adhesive materials, also called pressure-sensitive adhesives (PSA's), are special adhesive materials designed to stick on almost any surface by simple contact under light pressure [1]. This class of adhesives does not experience any physical transformation or chemical reaction during the bonding process. Commonly two different types of PSA's are produced depending on the components employed to produce them, these are, either Solvent Borne or Water Borne adhesives. Solvent borne PSA's provide excellent moisture resistance and good wetting properties. However, due to environmental problems, a shift from solvent borne to water borne type has been taking place.

One way to produce water-borne, environmentally friendly hybrid polymers that synergistically combine the positive properties of different materials is by means of the use of miniemulsion polymerization technique $[2,3,4]$. In particular, this technique has been employed to incorporate polyurethanes polymer chains into acrylic polymers in an attempt to improve the adhesive properties of the acrylic waterborne pressure-sensitive adhesives. The so obtained hybrid materials are found to have adhesive properties that are determined by the polymer architecture. Due to the interest of these hybrid materials, studying their structure, their chemistry and also their physical properties has been the main subject of several reports $[5,6,7]$. In this context, the hybrid Acrylic-Polyurethane (Acrylic/PU) polymers synthesized via miniemulsion photo-polymerization have been reported to be good candidates as pressure sensitive adhesives [8,9]. With the acrylic part as the majority component and PU chains used to form a network, it has been reported that small changes in the formulation and synthesis conditions of these materials give rise to rather different adhesive properties [10]. However, the ultimate reasons for the observed differences remain unclear.

In this work we demonstrate that dielectric relaxation techniques provide a complementary way of characterizing these hybrid materials, which will eventually help in establishing the characteristics at the origin of the material adhesives properties. Particularly, we have focus our attention on the dielectric study of the components of a hybrid Acrylic/PU pressure sensitive adhesive latex. This latex is composed by a GEL fraction and a SOL fraction that coexist within each miniemulsion droplet. Moreover, a pure acrylic polymer synthesized in a similar way has been also investigated as a reference 
of the majority acrylic component $(\approx 90 \%)$ in the latex.

\section{Experimental}

A complete description of the materials used and the synthetic methods employed at the fabrication of the hybrid Acrylic/PU PSA's was described elsewhere [8, 10]. In short, 2-ethylhexylacrylate (2-EHA), n-octadecylacrylate (SA), methyl methacrylate (MMA), methacrylic acid (MAA) and 2-hydroxy ethyl methacrylate (HEMA) acrylic monomers (2-EHA/ SA/ MMA/ MAA/ HEMA, 91.5/5.8/1.4/0.9/0.4 wt/wt) were mixed with a (10 wt\%) isocyanate terminated polyurethane (PU) prepolymer (see Fig. 1), Incorez 701 (PU, $3285 \mathrm{~g} / \mathrm{mol}$ ) (Incorez Ltd.), Bisphenol A (BPA) chain extender, dibutyltin dilaurate polyaddition catalyst and the oil soluble photoinitiator 1-hydroxy cyclohexyl phenyl ketone (I-184). This organic mixture was then mixed with the aqueous phase containing the surfactant, Dowfax 2A1, followed by a sonication step to obtain the miniemulsion. The so obtained miniemulsion was then photopolymerized in a continuous quartz tubular reactor placed in a UV chamber at $25^{\circ} \mathrm{C}$, with an incident light irradiance of $3.5 \mathrm{~mW} / \mathrm{cm}^{2}$. As mentioned above, the resulting product is a latex composed of a GEL fraction and a SOL fraction that coexist within each miniemulsion droplet. The GEL fraction is composed of a network of linear acrylic chains with PU chains attached to the reactive sites of the main acrylic backbone through bifunctional HEMA monomer [11]. These PU chains link the acrylic backbones together to form the reticulated structure. The SOL fraction is made of acrylic polymer chains with polyurethane pendants attached to the main acrylic backbone. The SOL and the GEL part of the polymer were separated by Soxhlet extraction under THF reflux. A representative drawing of the GEL network and the SOL fraction is presented in Fig. 1. A material synthetized in similar conditions to obtain only acrylic chains, polyethylhexylacrylate (PEHA), without PU nor BPA, was used as reference and it is also schematized in the same figure. It was seen that both the only acrylic polymer and the SOL fraction of the composite polymer had a similar average molecular weight of about $3 \times 10^{6} \mathrm{~g} / \mathrm{mol}$.

When preparing the samples for different experimental setups, all were first stored at $80^{\circ} \mathrm{C}$ in a vacuum oven for twelve hours. After this period of time the samples were allowed to cool down to room temperature in the vacuum oven before placing them into the corresponding sample holders. Before 


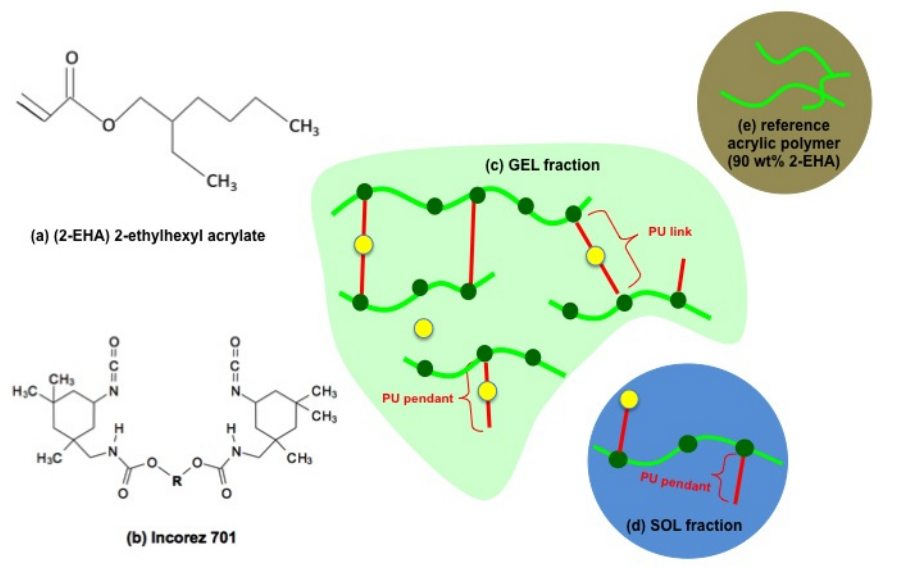

Figure 1: Chemical structural formula of (a) 2-ethylhexyl acrylate, (b) PU pre-polymer Incorez 701 and schematic representation of (c) GEL network, (d) SOL fraction and (e) acrylic reference polymer (PEHA). Green solid lines represent acrylic chain backbones, the green darker dots over the green lines correspond to junction points along the backbone, red lines represent PU chains and yellow circles correspond to BPA chain extenders.

the experiments were performed, in situ heating up to $150^{\circ} \mathrm{C}$ of the samples was conducted to remove any trace of moisture and/or solvent residues.

\section{Techniques}

\subsection{Differential Scanning Calorimetry (DSC)}

The experiments were carried out using a Q2000 set up from TA Instruments with a liquid nitrogen cooling system using $25 \mathrm{~mL} / \mathrm{min}$ helium flow. Temperature-modulated experiments were conducted using $60 \mathrm{~s}$ period and $0.5 \mathrm{~K}$ in amplitude, during cooling and heating at a mean rate of $3 \mathrm{~K} / \mathrm{min}$, in the temperatures range $150-420 \mathrm{~K}$. Samples for DSC experiments were prepared after the thermal treatment mentioned above, by encapsulating $5-10 \mathrm{mg}$ of sample in aluminium pans.

\subsection{Broadband Dielectric Spectroscopy (BDS)}

Measurements of the frequency dependent complex dielectric permittivity $\left(\epsilon^{*}(f)=\epsilon^{\prime}(f)-i \epsilon^{\prime \prime}(f)\right)$ were performed in the range $f: 10^{-1}-10^{6} \mathrm{~Hz}$, using a Novocontrol high-resolution dielectric analyser. The sample capacitor preparation for this setup consisted in an upper gold-coated electrode of 
$20 \mathrm{~mm}$ placed on a prepared film of the sample over a $30 \mathrm{~mm}$ gold-coated electrode. A separation of $100 \mu \mathrm{m}$ between both electrodes was maintained by using a cross-shaped Teflon spacer of small area. The sample cell was set in a cryostat, and its temperature was controlled via nitrogen gas jet stream coupled with the Novocontrol Quatro controller. The isothermal dielectric experiments were performed while cooling the sample from 420 to $150 \mathrm{~K}$ in steps of $10 \mathrm{~K}$ typically. The data reproducibility was checked during subsequent heating from 150 to $410 \mathrm{~K}$. For the BDS analysis, the main part of the measured dielectric losses was fitted according to the empirical HavriliakNegami (HN) equation [12]:

$$
\epsilon_{H N}^{*}=\epsilon_{\infty}+\frac{\Delta \epsilon}{\left(1+\left(i \omega \tau_{H N}\right)^{\alpha}\right)^{\beta}}
$$

In this equation, $\omega=2 \pi f, \alpha$ and $\beta$ denote respectively the symmetric and asymmetric broadening of the relaxation loss peak $(0<\alpha, \alpha * \beta \leq 1), \Delta \epsilon$ is the relaxation strength, $\epsilon_{\infty}$ the high frequency limiting permitivity and the $\mathrm{HN}$ relaxation time, $\tau_{H N}$, related to the loss peak relaxation time, $\tau_{\max }=$ $1 /(2 \pi f)$ by the equation:

$$
\tau_{H N}\left[\sin \left(\frac{\alpha \pi}{2 \beta+2}\right)\right]^{1 / \alpha}=\tau_{\max }\left[\sin \left(\frac{\beta \alpha \pi}{2 \beta+2}\right)\right]^{1 / \alpha}
$$

\section{Results}

As a first result we show the Differential Scanning Calorimetric (DSC) measurements reflecting the thermal properties of the polymers under study. No signatures of crystallization and/or melting were detected in the explored temperature range (up to $470 \mathrm{~K}$ ). Thus, in Fig. 2 we present three different curves corresponding to the reversing heat capacity as a function of the temperature for all samples understudy, GEL, SOL and PEHA.

The data were obtained while cooling the material from $420 \mathrm{~K}$ at a rate of $3 \mathrm{~K} / \mathrm{min}$. The three samples explored by DSC exhibit similar thermal behaviour, particularly, at the glass transition where all of them exhibit a clear step like feature at about $210 \mathrm{~K}$. It can be seen that the curve of the reference acrylic sample (PEHA) seems to exhibit a small but noticeable shift towards lower values of the glass transition temperature (measured as the temperature at the middle of the step) by about $2 \mathrm{~K}$. In addition, we notice that SOL sample exhibits an additional small "step-like" change at 


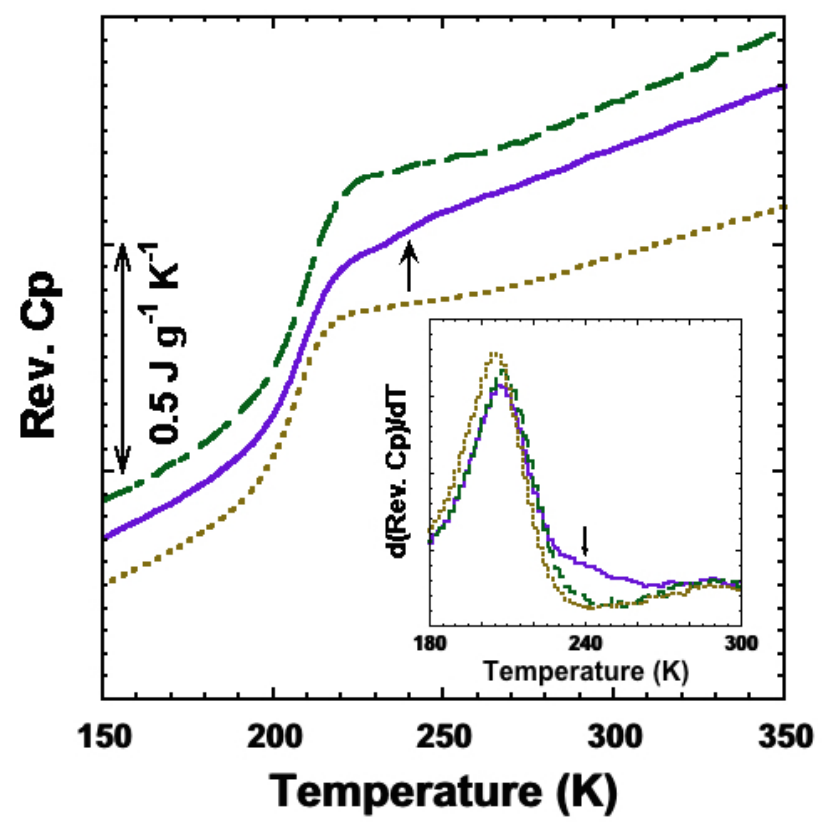

Figure 2: Differential Scanning Calorimetric scans for three different samples; GEL fraction of the obtained latex (solid line), soluble part of the latex, named SOL (dashed line), and the reference acrylic polymer, PEHA (dotted line). Inset in the figure corresponds to the derivative with respect to temperature of the reversible specific heat for the three samples.

about $240-250 \mathrm{~K}$, a feature that is absent in the other two samples. These differences are more evident in the inset of Fig. 2 where the temperature derivative of the curves are depicted.

The main objective of this work was to investigate the dielectric relaxation on these hybrid polymers around and above the glass transition. Whereas the dielectric relaxation around the glass transition is known to be governed by the same molecular motions responsible of the thermal glass transition, large scale structures can be also detectable in the dielectric relaxation phenomena either by the existence of associated dipole moment fluctuations (normal mode dielectric relaxation originated by end-to-end vector fluctuations, mesogenic group reorientations in polymeric liquid crystals) and/or the effect of the interfaces on the charge transport (interfacial polarization phenomena) [12]. Fig. 3 presents the real (a) and imaginary (b) part of the dielectric permittivity as a function of temperature (isochronal representation) at the 
frequency of $1 \mathrm{~Hz}$ for the three samples.
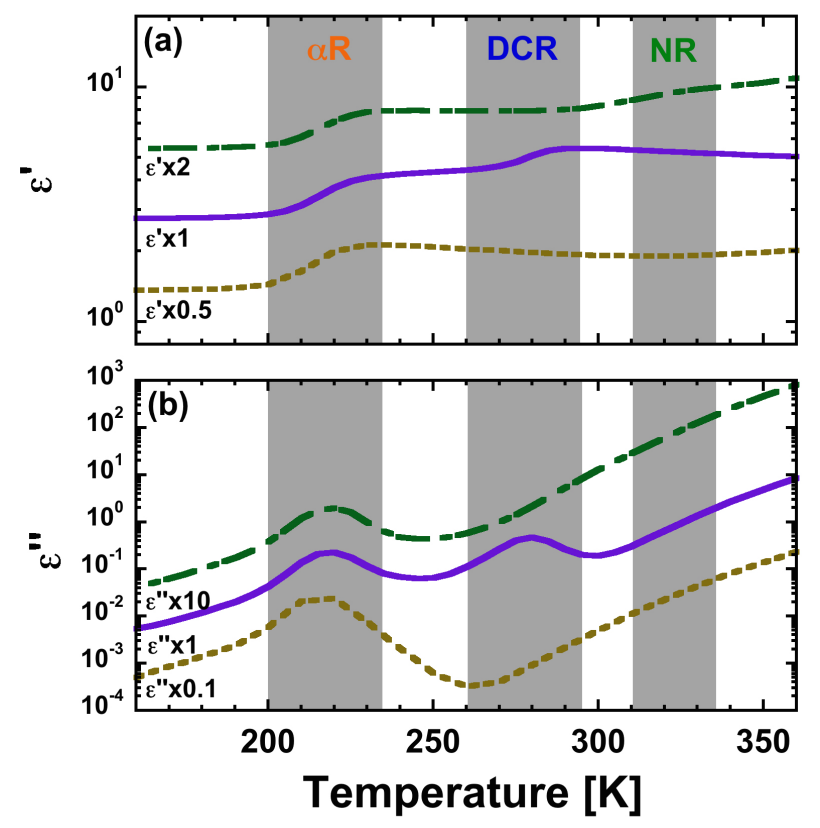

Figure 3: Real (a) and Imaginary (b) part of the dielectric permittivity as a function of temperature for GEL (dot-dash line), SOL (solid line), and the acrylic polymer, PEHA (dashed line), in the isochronal representation.The shadowed areas in the figure correspond to the three different temperature regions where the alpha relaxation $(\alpha \mathrm{R})$, dangling chain relaxation (DCR) and Network Relaxation (NR) are detected.

The first feature to notice in Fig. 3 is that we can distinguish three different temperature regions where dielectric relaxation processes are detected. If we focus our attention at the imaginary part of the dielectric data, that is Fig. 3 (b), we notice that the temperature region about $200-230 \mathrm{~K}$ is where the three samples present a clear dielectric relaxation. This is a similar temperature region to that where the glass transition is observed in DSC experiments. Therefore this dielectric relaxation has to be related to the segmental dynamics dominated by the majority acrylic monomeric units. We will identify this relaxation as the so-called $\alpha$-Relaxation $(\alpha \mathrm{R})$. The second temperature region where dielectric relaxation is detected is about $270-290 \mathrm{~K}$. Here SOL sample presents a prominent loss peak; without any signature of 
such relaxation in the reference acrylic sample. The major difference between SOL and PEHA samples is that the first contains PU chains attached to the main acrylic backbone by one side of the polyurethane chain (PU pendants), whereas the second one does not. Therefore this second relaxation process should be related to dipole moments given by the PU chains, and thus this relaxation will be referred as Dangling Chain Relaxation (DCR). Moving towards higher temperatures and examining the imaginary part of the dielectric data, Fig. 3 (b), show that in that region the d.c. conductivity is dominant. However, when analyzing the real part of the dielectric data in the same temperature region we notice that the GEL sample presents a step like feature about $320 \mathrm{~K}$. This feature is not observed for SOL neither for PEHA samples. Thus, this third relaxation process would be linked to presence of a network structure in the GEL sample and so it will be called Network Relaxation (NR).

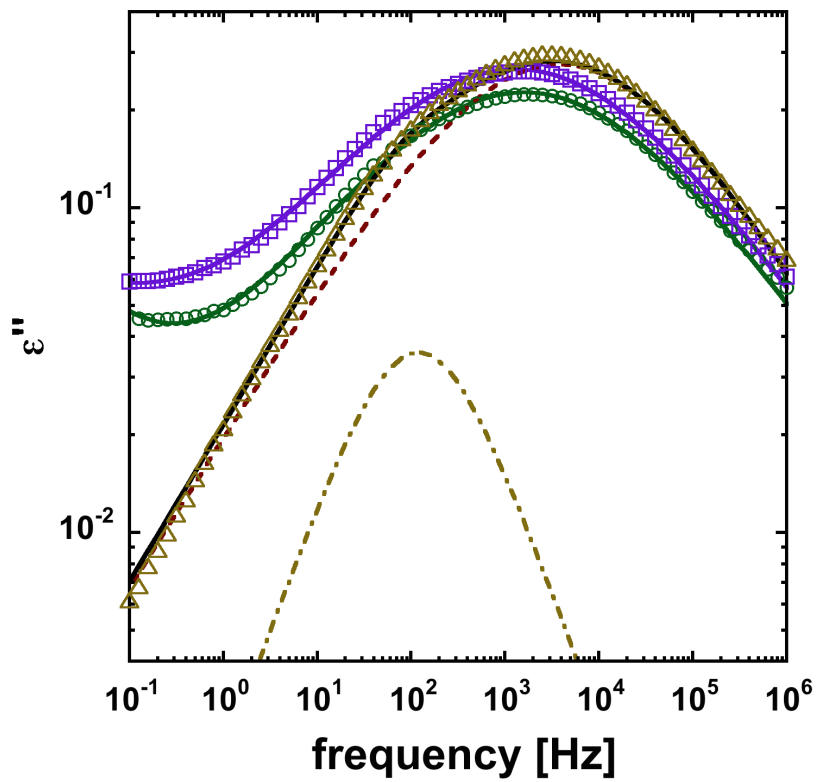

Figure 4: Frequency dependent Imaginary part of the dielectric permittivity at $240 \mathrm{~K}$ of GEL (circles), SOL (squares) and reference acrylic polymer (PEHA) (triangles). Lines correspond to the fitting of the reference acrylic data to the sum of two Cole-Cole functions, dotted and dash dotted lines representing the components and solid line the sum.

As we showed above, the $\alpha \mathrm{R}$ process is observed in the three samples stud- 
ied. In Fig. 4 we present the imaginary part of the dielectric permittivity as a function of the frequency for the three different samples at a temperature of $240 \mathrm{~K}$, where the $\alpha$-relaxation process is clearly observed. As already commented, these loss peaks would be related to the segmental mobility involving polar groups of the acrylic chains explaining why the relaxation process exhibits similar characteristics, such as the intensity of the peak maxima and peak shape and position, for the three samples. One clear difference of the GEL and SOL samples with respect to the reference acrylic polymer, is that in the low frequency range the formers exhibit a plateau like behaviour with relatively high loss values, while the latest show a monotonous power-law like change of the losses. The higher intensity in GEL and SOL samples is indicative of the existence of slower contributions likely due to motions involving the PU chains segments. As the reference acrylic sample does not contain such PU chains, in order to obtain an accurate description of the $\alpha$ relaxation process we preferred starting our analysis with the dielectric data obtained for the reference acrylic sample (PEHA). The $\alpha$-relaxation process was modelled by the sum of two Cole-Cole [12] functions (Eq. 1, with $\beta=1$ ) to take into account not only the main relaxation, but also a slower and weak contribution detectable as a shoulder that could be related to the presence of a minor amount of hydroxyl groups as part of the acrylic chains. It is important to clarify that due to the weakness of this relaxation, in the final fitting (see Fig. 4) at all temperatures, both the shape parameter and relaxation strength of the weak relaxation component were fixed to the average parameter values obtained in a preliminary unrestricted fitting. Fig. 4 shows that fitting in this way the loss data of the reference acrylic polymer are very well described.

When fitting the dielectric $\alpha$-relaxation of the SOL and GEL samples we used an approach similar to that used for the reference sample but with further constrains. Namely, these two samples evidence the presence of extra contributions at lower frequencies that would be related with the presence of the PU chains. This prevents an accurate characterization of the weak component of the $\alpha$-relaxation in these two samples and consequently we have restricted the fitting by imposing constrains to the three parameters describing the weak component. Namely, the ratios between both the relaxation times and relaxation strengths of these two components as well as the shape parameter of the weaker were fixed to those describing the reference polymer. This is justified by the assignment of this weaker component to minor molecular groups that would be in any case related with the acrylic 


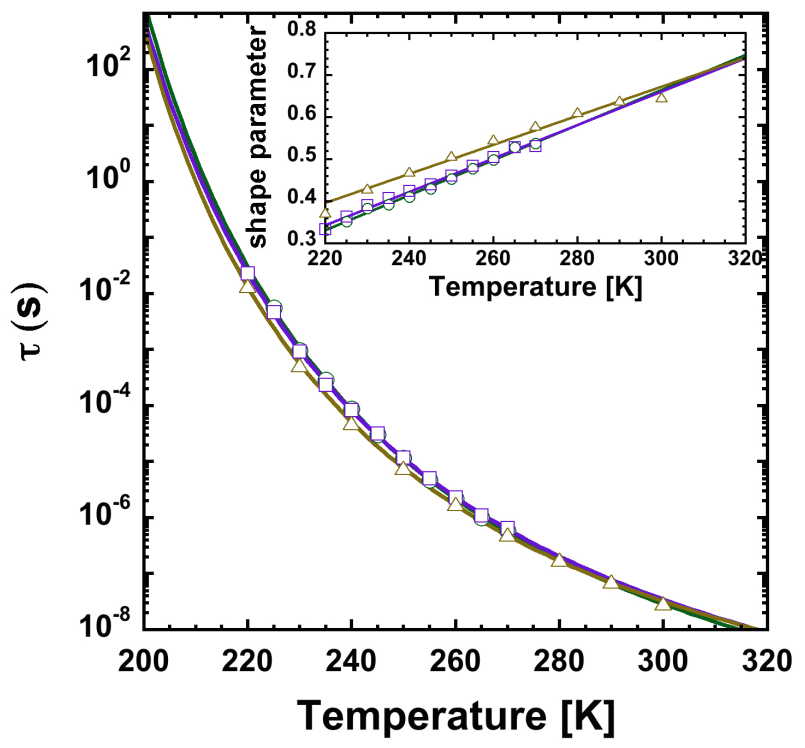

Figure 5: Characteristic relaxation times for GEL (open circles), SOL (open squares), and the reference acrylic polymer (PEHA) (open triangles). The lines in the figure correspond to VFT fitting.

main chain and consequently both components are expected to change in a similar way when incorporating the PU branching chains. Furthermore, a power law tail-like contribution was added to account for the mentioned extra low frequency losses, with a fix exponent value -0.15 , which was found adequate for describing the data of SOL sample at $300 \mathrm{~K}$ in this intermediate range (see below). As can be seen in Fig. 4, in this way a very good fitting is obtained by allowing only four parameters to vary, namely the relaxation time, shape and strength of the main component of the $\alpha$-relaxation and the amplitude factor of the power law contribution.

The similarities in the $\alpha$-relaxation range for the three samples observed in Fig. 4 are confirmed in a more quantitative way by comparing the temperature dependence of the main relaxation parameters so obtained. Particularly, the characteristic relaxation times $\left(\tau_{\max }\right)$ for the three samples, GEL, SOL and PEHA, (see Fig. 5) exhibit typical super-Arrhenius behaviour with minor differences that could be straightforwardly associated to the small changes in the glass transition temperatures as detected by DSC. For a quantitative probe of that, we have fitted the data by using the Vogel-Fulcher-Tammann 
(VFT) equation $[13,14,15]$. The VFT equation reads as:

$$
\tau=\tau_{\infty} \exp \left(\frac{D T_{0}}{T-T_{0}}\right)
$$

where the value of $\tau_{\infty}$ has been fixed to one ps., which is in the range of the time corresponding to the molecular vibrations, $T_{0}$ is the so-called Vogel temperature, and $D$ is a dimension-less parameter often related to the so called kinetic fragility index. Solid lines in Fig. 5 correspond to the main relaxation process of the three samples, the lines fitting the data of the main relaxation component correspond in the three samples to VFT equations with fixed values of $D=9.4$ and $T_{0}$ values changing only slightly from sample to sample. Particularly we obtain: $T_{0 G E L}=158.3 \mathrm{~K}, T_{0 S O L}=158.1 \mathrm{~K}$ and $T_{0 P E H A}=156.9 \mathrm{~K}$. On the other hand, when comparing the VFT parameters of the weak component with those of the main one, it is found that the parameter $D$ is much higher $(D=18)$, and conversely, the value of $T_{0}$ resulted significantly lower $\left(T_{0}=129 \mathrm{~K}\right)$. Noteworthy, both time scales nearly match each other at $T_{g}$.

The inset in Fig. 5 shows the shape parameters of the main relaxation component as a function of temperature for the three samples. It is found that in the reference polymer this parameter is systematically higher (a slightly narrower peak) than in the SOL and GEL samples. However, in all cases the shape parameter increases with the temperature and the values at the highest temperatures become indistinguishable. For the relaxation strength we obtain also slightly higher values for the reference acrylic polymer, which could be expected because the $\approx 10 \%$ reduction in the acrylate group density due to the incorporation of the PU chains. Nevertheless, the differences among samples are close to the typical uncertainties of this parameter $(\approx 10 \%)$ originated by the difficulties in the determining accurately the actual sample capacitor geometry. Namely we found: $T * \Delta \epsilon_{G E L}=280 \mathrm{~K}$, $T * \Delta \epsilon_{S O L}=320 \mathrm{~K}$ and $T * \Delta \epsilon_{P E H A}=360 \mathrm{~K}$.

Summarizing the relaxation results, the analysis done shows that the segmental relaxation process is made by sum of two detectable relaxation processes; one weak component and a major one that exhibit similar dielectric properties for the three samples under study.

Next, we consider the second relaxation process (DCR) that it is very pronounced in the SOL sample, i.e., the soluble part of the latex obtained, where most of the PU chains are attached to the acrylic polymeric backbone by a single end, as side chains. Fig. 6 shows the imaginary part of the dielec- 


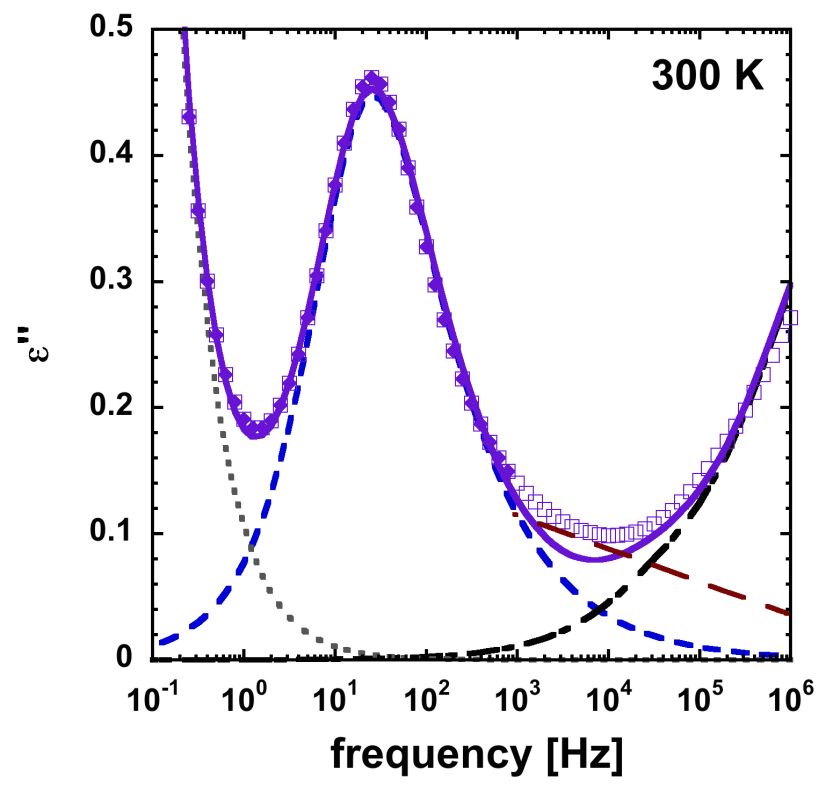

Figure 6: Imaginary part of dielectric permittivity as a function of frequency for SOL (squares) sample. In the figure dotted line corresponds to the conductivity, the shortdash and dot-dash lines correspond to the fitting of the data to a Havriliak-Negami and the extrapolated model for $\alpha \mathrm{R}$ respectively. Solid line corresponds to the whole losses description including also the conductivity contribution. Long-dashed line corresponds to a power law description with exponent -0.15 of the intermediate range (see the text).

tric data as a function of the frequency at a temperature of $300 \mathrm{~K}$, where a well-defined and asymmetric loss peak is observed. At first, these data were fitted with a Havriliak-Negami equation as the main component (short-dash line in Fig. 6). However, in the fitting routine we also included two additional contributions. The low frequency tail increase was modelled as due to the d.c. conductivity (dotted line), i.e., $\epsilon_{0} \epsilon^{\prime \prime}=\sigma_{d c} / \omega$. The high frequency range still exhibits the contributions corresponding to the $\alpha$-relaxation that was modelled extrapolating the parameters obtained for this sample at lower temperatures (dot-dash line). Nevertheless, when using this procedure, there was a mismatching of the fitting function (solid line) and the data at frequencies of about $10^{3}-10^{4} \mathrm{~Hz}$. This is indicative of some extra minor contribution that make the measured losses in this range to behave as a power law tail (see long-dashed line), which was used above in describing the lower temperature 
data at low frequencies. Because of that, the fitting parameters reported below were obtained by considering only the loss data points fulfilling the condition $0.1<\epsilon^{\prime \prime}(\omega) / \epsilon_{\max }^{\prime \prime} \leq 1$, where $\epsilon_{\max }^{\prime \prime}$ refers to the loss value at the peak frequency. In fitting these data we used the sum of two contributions, a HN relaxation function and a dc-conductivity component.

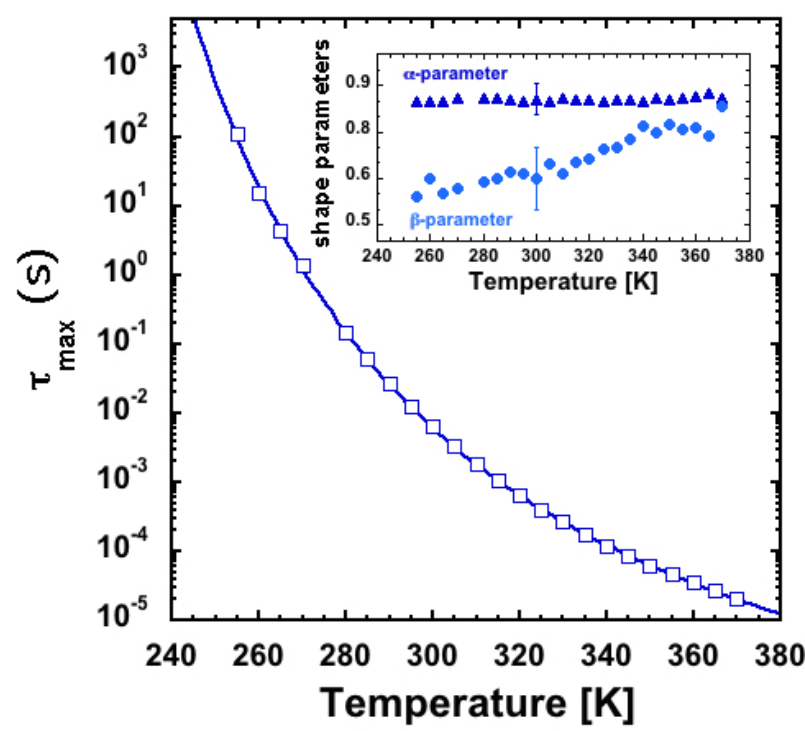

Figure 7: Loss peak relaxation times for the dangling chain relaxation. The line in the figure represents the VFT fit to the relaxation times. Inset shows the shape parameters of this relaxation process with representative error bars.

Fig. 7 show the so obtained values of the loss peak relaxation time as a function of the temperature. The inset in the figure shows the corresponding relaxation shape parameter values. When the VFT equation is used to fit the data it is found that, on one hand the pre-exponential factor of the DCR times is quite large $(\approx 1.5 \mathrm{~ns})$ suggesting that the molecular unit responsible for this relaxation is also large. On the other hand, the value of $T_{0}(184 \mathrm{~K})$ is much higher than that of the $\alpha$-relaxation. Noteworthy, the obtained value of $D$ is 9.56, that is, nearly indistinguishable from that of the $\alpha$-relaxation.

Concerning the shape of the DCR, inset in Fig. 7 shows that the loss peak is less asymmetric as temperature increases becoming relatively narrow at the highest temperatures. Finally, we found that the relaxation strength can be well approximated as: $T * \triangle \epsilon_{D C R}^{S O L}=420 \mathrm{~K}$. i.e. about $15 \%$ larger 
than that of the $\alpha$-relaxation at a similar temperature.

The third dielectric relaxation process (NR) is detectable only in the GEL sample at higher temperatures. This strongly suggests that it is related with the network structure, but its actual molecular origin is unclear. A detail analysis of this NR should help to this end.

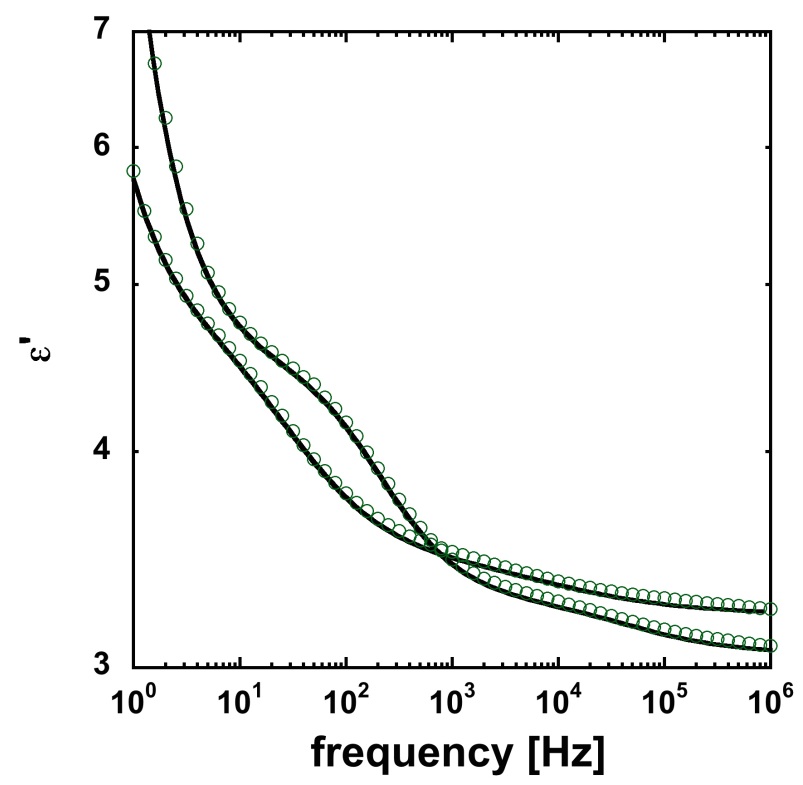

Figure 8: Real part of the dielectric function for GEL sample at $370 \mathrm{~K}$ and $410 \mathrm{~K}$. Solid lines correspond to the fitting model.

Fig. 8 shows the real part of the dielectric permittivity of the GEL sample as a function of frequency at two temperatures. Here, we can clearly identify a step like feature corresponding to the network relaxation process. However, the direct evaluation of the low frequency plateau determining the static permittivity value is prevented by an upward of the measured values, most likely due to the onset of interfacial polarization effects. Interfacial polarization arises when the conductivity of the material filling the sample capacitor increases so much that significant accumulation of ions occurs in internal sample interfaces (commonly known as Maxwell-Wagner-Sillars relaxation) [12] and/or in the contact between the sample and the electrodes. It is clear that at these high temperatures (more than $150 \mathrm{~K}$ above $T_{g}$ ) the conductivity of the samples is relatively high. 


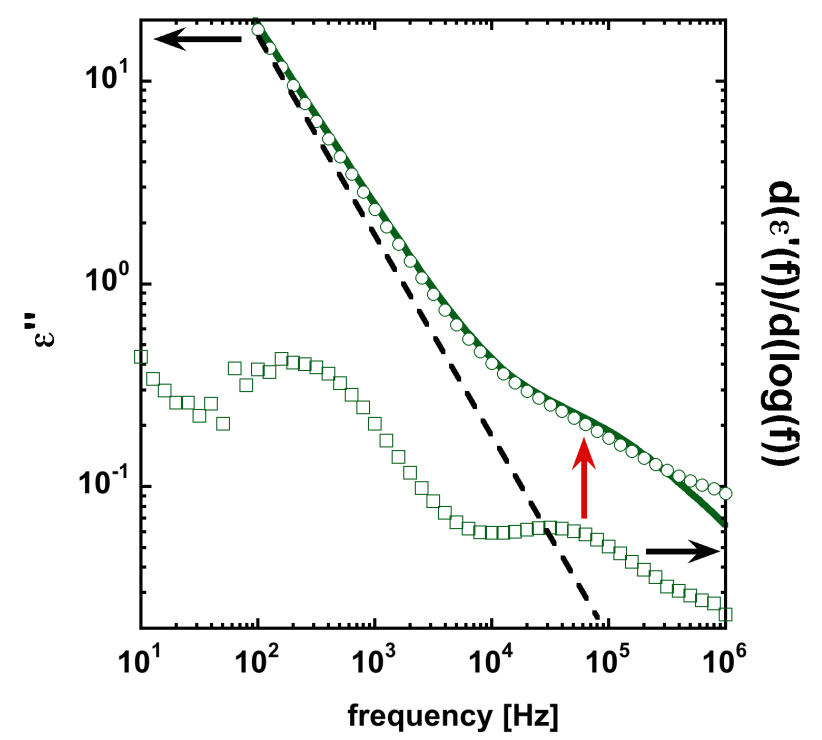

Figure 9: Imaginary part of the dielectric function as a function of frequency for GEL at $410 \mathrm{~K}$. Solid points correspond to the measured loss data and open squares correspond to the derivative of the real part with respect to the logarithm of frequency. The arrow exhibits the peak position of the SOL sample at the same temperature. The dashed line corresponds to the dc conductivity contribution.

In order to gain more information on the NR we will also analyse the imaginary part of the dielectric data of GEL at this temperature, circles in Fig. 9, despite the fact that the conductivity contribution is by far dominating the low frequency range (see line in Fig. 9). The network relaxation phenomenon in this representation is not easily detectable, as the data do not exhibit any visible feature in the corresponding frequency range since the signal is dominated by the dc conductivity contribution. However, we can clearly distinguish a small shoulder appearing at higher frequencies, of about $10^{5} \mathrm{~Hz}$ at $410 \mathrm{~K}$. Thus, to identify more clearly the relaxation processes detectable in the GEL sample at this temperature, we have made use of one-dimensional derivative techniques $[12,16]$, namely we have calculated numerically the first derivative of the real part of the dielectric permittivity with respect to the logarithm of the frequency (squares in Fig. 9). In this derivative representation both relaxation features become evident, a rather prominent peak is detected around $300 \mathrm{~Hz}$ and a weaker but well resolved 
one at about $400 \mathrm{kHz}$. Moreover, by comparing these data with those obtained at the same temperature for the SOL sample it seems that the weak relaxation detected in the GEL can be related with the DCR identified above (see vertical arrow in Fig. 9).

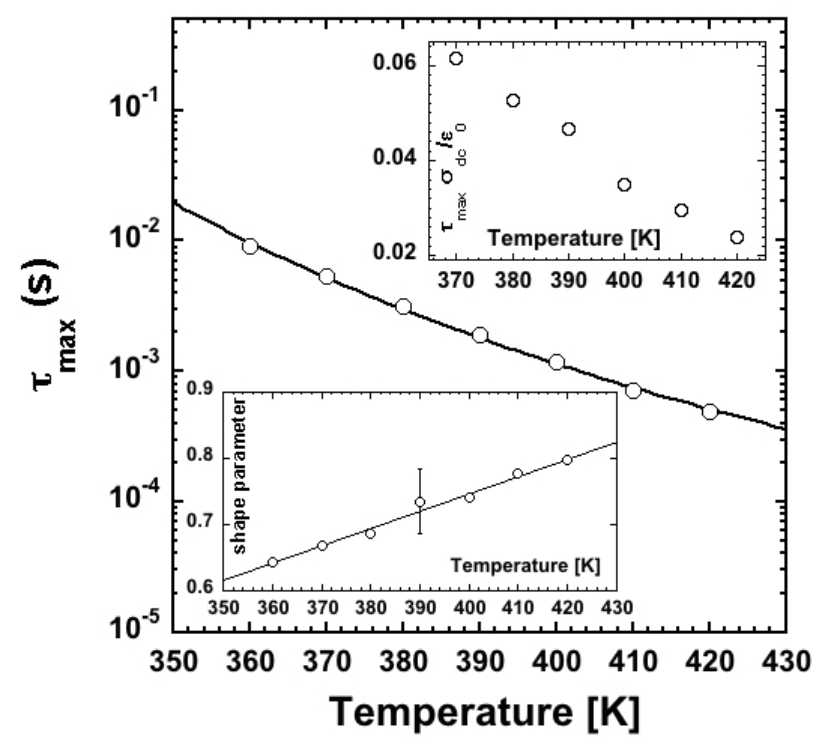

Figure 10: Characteristic relaxation times for the network relaxation process. The line in the main frame represents the VFT fit to the relaxation times. Lower inset shows the shape parameter of the network relaxation described by a linear temperature variation (line) with a representative error bar. Upper inset evidences the distinct temperature dependence of the relaxation time and the sample dc conductivity.

The quantitative analysis of the GEL sample data was made by fitting both the real and imaginary parts of the dielectric data to the superposition of two symmetric Cole-Cole equations plus a d.c. conductivity contribution and an additional low frequency power law increasing of the real part. The peak time of the weak DCR component in the GEL sample was fixed to that obtained in the SOL one. This method was applied to temperatures where the NR component is still well resolved from the low frequency increase in the real part, i.e. for $T>350 \mathrm{~K}$. Lines in Fig. 8 and 9 show representative examples of this approach, evidencing that in this way we obtained a high quality fitting, maintaining a moderate uncertainty in the parameters characterizing the NR of the GEL sample. The results so obtained for the NR time of the GEL 
sample are presented in Fig. 10. The lower inset in Fig. 10 shows that the NR relaxation narrows gradually by increasing temperature. By analysing the temperature dependence of the NR times we have found that a VFT equation with either $T_{0}=184 \mathrm{~K}$ (that found for DCR) or $T_{0}=158 \mathrm{~K}$ (that found for $\alpha \mathrm{R}$ ) describe equally well the data. The corresponding values of $D$ and $\tau_{\infty}$ are 11 and $90 \mathrm{~ns}$, and 16 and $25 \mathrm{~ns}$, respectively. Even if the uncertainty is large, the values of the pre-exponential factors are in the range of tens nanosecond, i.e. motions much slower than those of the DCR suggesting molecular entities considerably larger than those responsible of the DCR. On the other hand, it is found that the relaxation strength of the NR is given in good approximation by $T * \Delta \epsilon_{N R}=555 \mathrm{~K}$, which again is quite large taking into account the relatively low amount of PU chains.

The results presented above evidence that despite the similar thermal behaviour exhibit by the three acrylic based polymers investigated, they present strong differences in the dielectric relaxation around and above room temperature. Particularly, the SOL and GEL fractions of the hybrid Acrylic/PU material obtained by incorporating about $10 \%$ PU pre-polymer to the polymerization reaction of the acrylic units present prominent characteristic relaxations in addition to the more conventional relaxation connected with the glass transition phenomenon. The SOL sample shows a well-resolved loss peak, with a maximum frequency at about $100 \mathrm{~Hz}$ at room temperature, and the GEL sample presents a distinguishable slower relaxation reaching the same peak frequency of $100 \mathrm{~Hz}$ at about $100^{\circ} \mathrm{C}$. The fact that in these two samples there are additional PU chains either as side chains of the main acrylic ones or as bridges forming a network (in the GEL sample only) naturally leads to relate this new relaxation processes with the presence of the PU chains and look for the molecular origin of the resolved dielectric relaxation in connection with the distinct polymer architectures. Contrary the $\alpha$-relaxation associated to the glass transition is quite similar in all the samples, which clearly indicates that it is mainly determined by the segmental mobility of the acrylic units. These results opens the possibility of using the dielectric relaxation as a tool for characterization of this kind of hybrid Acrylic/PU materials provided the molecular origin of the relaxation processes is properly addressed. 


\section{Discussion}

In this context, the result obtained on the SOL sample seems to unambiguously evidence that the slower relaxation detected cannot be originated by interfacial polarization phenomena, but must be attributed to the dynamics of the PU chains attached to the main acrylic ones. The reason is that the low conductivity of the sample in the relevant temperature range ensures that the dielectric relaxation detected is due to some molecular dipole fluctuation. Furthermore, it is expected that the presence of PU chains in this sample should necessarily result in higher values of the static permittivity because the incorporation of quite strong molecular dipoles. Since there is no hint of contribution of such additional dipoles to the dielectric $\alpha$-relaxation (the $\alpha$ relaxation strength decreases a bit with respect to the reference only acrylic sample) the one detected at higher temperatures/lower frequencies must be originated by these PU chains. When considering the specific characteristics of this relaxation process it is very significant the fact that the relaxation time changes with temperature according with a VFT equation with a preexponential factor much higher than the reciprocal of the typical vibrational frequencies. This implies that the molecular dipole would involve a relatively large molecular entity. For instance, this is the case of the terminal relaxation in polymers that in some cases is also detectable dielectrically as the so-called normal mode relaxation. This dielectric relaxation is the result of the fact that in these polymers (1,4 cis-polyisoprene as a prototype) [17] there is a dipole moment proportional to the end-to-end chain vector and therefore a detectable dielectric relaxation reflecting these fluctuations. However, there is not evidence of the presence of an end-to-end dipole moment in PU polymers. Moreover, if any, the strong dielectric strength and quite narrow loss peak DCR would be hardly compatible with this origin. Note particularly that the time scales of a normal mode depends as a power of the chain mass with the exponent 3 for untangled chains, and 3.4 for larger chains, so only for very monodisperse polymers a narrow dielectric loss peak can be found.

On the other hand, it has already been widely reported [18, 19, 20, 21] that the so called segmented polyurethanes are typical block copolymers with a block consisting of a relatively flexible soft segment (SS) and the other block forming a highly polar hard segment (HS). This structure usually leads to microphase separation (MS) at mesoscopic length scales, resulting in a morphology consisting of microdomains rich in each of the blocks. The DSC results of the SOL fraction (see Fig. 2) had evidenced the presence of a 
weak jump of the heat capacity at around $240 \mathrm{~K}$ (30 K above the main glass transition) that could be indicative of the occurrence of some PU segregation in this sample. This opens the possibility to associate the observed dielectric relaxation to this segregated phase. Taking into account the temperature where the DCR is detectable $\left(\tau_{D C R}=1 \mathrm{~s}\right.$ at $T \approx 270 \mathrm{~K}$ as seen in Fig. 5), it cannot be directly associated to the glass transition phenomenon within this phase. Contrary, as aforementioned, the properties of the DCR point to a bigger scale motion.

It is well known that PU segments have a strong tendency to crystallize [22]. The investigated samples show no signature of crystallization, but this circumstance can favour the development of some orientational correlation [23]. So, a suitable origin of the DCR could be the presence of certain kind of mesogenic order in the segregated phase, which would be compatible with the relatively large molecular entity responsible for the DCR. Moreover, the existence of mesogenic-like entities with relatively strong dipole moment can result in a significant dipole orientation correlation. This scenario could explain the quite strong DCR strength observed experimentally that arises due to just $10 \%$ of PU pre-polymer, which was added in the synthesis formulation. However, one question that remains to be clarified in this picture is whether a dielectric signature of the glass transition in the segregated phase exists. To answer this, we performed Thermally Stimulated Depolarization Current (TSDC) experiments $[12,24]$ which confirmed the presence of a distinguishable peak in the depolarization current at around $235 \mathrm{~K}$ just in between the two main peaks related respectively with the main $\alpha$-relaxation at $208 \mathrm{~K}$ and the DCR at $255 \mathrm{~K}$. Such a weak additional dielectric relaxation component will also be the responsible of the miss fitting of the SOL sample loss data shown in Fig. 6 in the valley (at around $10^{4} \mathrm{~Hz}$ ) in between the two main loss peaks. In this framework, the observation of a DCR component also in the GEL sample would be due to the existence of residual PU chains attached by a single end to the acrylic main chain without being involved in the network formation

The assignment of the slowest relaxation detected in the GEL sample is less straightforward. A main reason is that in the relevant temperature range the sample conductivity is relatively large, which can give rise to relaxation processes reflecting charge trapping at interfaces. Trying to test this possibility we have analysed the temperature dependence of the dc conductivity and compared it with that of the NR time. The upper inset in Fig. 10 shows that the quantity $\tau_{N R} \sigma_{d c} / \epsilon_{0}$ changes markedly with temperature, opposite to 
the expectation of simple models of dielectric relaxation associated to charge trapping [12]. On the other hand, the shape of the relaxation associated to charge trapping would be determined by the spatial distribution of the trapping interfaces and it would not be expected to change significantly with temperature. However, our finding is a marked decrease of the NR when increasing temperature. Overall, these results point to the fact that the NR is not originated in a charge trapping process but it would reflect the fluctuations of some molecular dipolar entity. Since the $\alpha$-relaxation in the GEL is also quite similar to that of the pure acrylic polymer, these molecular entities should involve the PU chains that in the GEL are cross-linking chains responsible for the network formation.

The differences respect to the DCR would be related with the fact that both PU chain ends are attached to the main acrylic backbone. This network structure would, on one hand, restrict the possibility of the PU units to form a well-segregated phase and on the other hand it would slow down the corresponding dipole moment fluctuations. Moreover, it is known that most of the PU chains forming the network have been extended during the synthesis process [9] (see scheme $\mathrm{c}$ in Fig. 1). This together with the required fluctuations of the network junction points would results in a large size of the molecular entities involved in the dipole moment fluctuations responsible of the NR. These could be bundles of PU hard segments forming part of the crosslinking chains with some degree of orientational order that would require the fluctuation of the network structure for a full decorrelation of the resulting dipole moments. A schematic representation of these bundles is depicted in NR-drawing of Fig. 11. In this drawing we also included some of the remaining PU chains attached by a single end that would be responsible for the weak DCR component in the GEL. Figure 11 shows a quantitative and qualitative summary of the three different dielectric processes studied in the Acrylic/PU samples together with schematic representations of the entities responsible for these relaxation processes.

\section{Conclusions}

In this work we show that Broadband Dielectric Spectroscopy (BDS) technique provide a suitable method for characterizing complex hybrid Acrylic/PU pressure sensitive adhesive materials. Despite the similar thermal behaviour of the three materials investigated, BDS results show up quite clear differences. We have been able to identify and establish the reason for these 


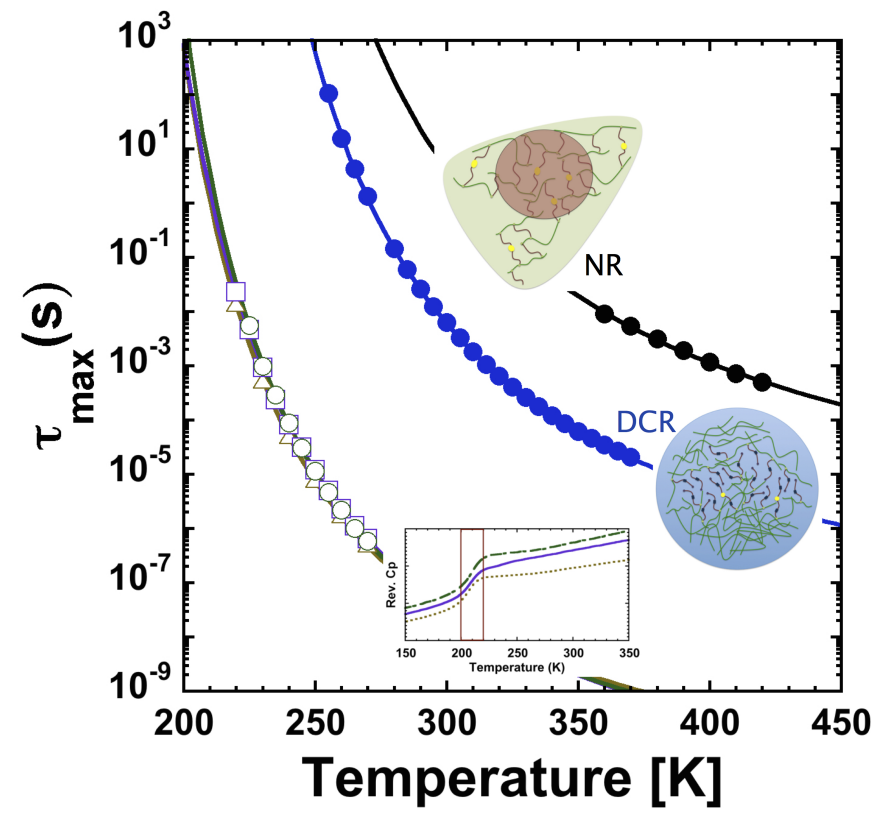

Figure 11: Characteristic relaxation times as a function of temperature for the three different relaxation processes $(\alpha \mathrm{R}, \mathrm{DCR}, \mathrm{NR})$ studied in these Acrylic/PU polymers. Correspondingly a schematic representation of entities responsible for the relaxation processes is depicted. 
differences on the basis of a molecular assignment of three main different relaxation processes. Particularly, the fastest of these relaxation processes is attributed to the segmental motion of the main acrylic chains $(\alpha R)$ and thus it exists in all samples. The analysis of the second fastest relaxation process that it is completely absent in the reference pure acrylic polymer and very prominent on the SOL sample it is attributed to the presence of some orientational correlations in the segregated PU phase. In this framework, the observation of a DCR component also in the GEL sample is attributed to the existence of residual PU chains attached by a single end to the acrylic main chain without being involved in the network formation. Concerning the slowest relaxation process (NR) that it is only present in the GEL sample, it has been attributed to the presence of orientationally correlated PU hard segment bundles of the PU chains forming the GEL network. A step forward in this direction, would lead us to the comparison (in particularly of the NR) between different GEL materials obtained by using the same chemical ingredients but varying synthetic conditions.

\section{Acknowledgements}

The authors gratefully acknowledge the financial support of the Basque Government codes: IT-654-13 and nanoIKER-2011-2013 (Etortek program) and also the Ministerio de Economía y Competitividad code:MAT2012-31088.

\section{References}

[1] Creton C. Pressure-sensitive adhesives: an introductory course. MRS bulletin 2003;28(06):434-9.

[2] Lopez A, Degrandi-Contraires E, Canetta E, Creton C, Keddie JL, Asua JM. Waterborne polyurethane- acrylic hybrid nanoparticles by miniemulsion polymerization: Applications in pressure-sensitive adhesives. Langmuir 2011;27(7):3878-88.

[3] Udagama R, Degrandi-Contraires E, Creton C, Graillat C, McKenna TF, Bourgeat-Lami E. Synthesis of acrylic- polyurethane hybrid latexes by miniemulsion polymerization and their pressure-sensitive adhesive applications. Macromolecules 2011;44(8):2632-42. 
[4] Wang C, Chu F, Graillat C, Guyot A, Gauthier C, Chapel J. Hybrid polymer latexes: acrylics-polyurethane from miniemulsion polymerization: properties of hybrid latexes versus blends. Polymer 2005;46(4):1113-24.

[5] Kyritsis A, Raftopoulos K, Rehim MA, Shabaan SS, Ghoneim A, Turky G. Structure and molecular dynamics of hyperbranched polymeric systems with urethane and urea linkages. Polymer 2009;50(16):4039-47.

[6] Kontou E, Spathis G, Niaounakis M, Kefalas V. Physical and chemical cross-linking effects in polyurethane elastomers. Colloid and Polymer Science 1990;268(7):636-44.

[7] Bur A, Fetters L. The chain structure, polymerization, and conformation of polyisocyanates. Chemical Reviews 1976;76(6):727-46.

[8] Daniloska V, Carretero P, Tomovska R, Paulis M, Asua JM. Highperformance adhesives resulting from spontaneous formation of nanogels within miniemulsion particles. ACS applied materials \& interfaces 2014;6(5):3559-67.

[9] Daniloska V, Tomovska R, Asua JM. Hybrid miniemulsion photopolymerization in a continuous tubular reactor?a way to expand the characteristics of polyurethane/acrylics. Chemical Engineering Journal 2012;184:308-14.

[10] Daniloska V, Carretero P, Tomovska R, Asua JM. High performance pressure sensitive adhesives by miniemulsion photopolymerization in a continuous tubular reactor. Polymer 2014;55(20):5050-6.

[11] Reyes Y, Lopez A, Asua JM. Modeling the microstructure of acrylicpolyurethane hybrid polymers synthesized by miniemulsion polymerization. Macromolecular Reaction Engineering 2011;5(9-10):352-60.

[12] Schönhals A, Kremer F. Analysis of dielectric spectra. In: Broadband dielectric spectroscopy. Springer; 2003, p. 59-98.

[13] Vogel H. The law of the relation between the viscosity of liquids and the temperature. Phys Z 1921;22:645-6. 
[14] Fulcher GS. Analysis of recent measurements of the viscosity of glasses. Journal of the American Ceramic Society 1925;8(6):339-55.

[15] Tammann G, Hesse W. Die abhängigkeit der viscosität von der temperatur bie unterkühlten flüssigkeiten. Zeitschrift für anorganische und allgemeine Chemie 1926;156(1):245-57.

[16] Wübbenhorst M, van Turnhout J. Analysis of complex dielectric spectra. i. one-dimensional derivative techniques and three-dimensional modelling. Journal of non-crystalline solids 2002;305(1):40-9.

[17] Lund R, Barroso-Bujans F, Slimani MZ, Moreno AJ, Willner L, Richter $\mathrm{D}$, et al. End-to-end vector dynamics of nonentangled polymers in lamellar block copolymer melts: the role of junction point motion. Macromolecules 2013;46(18):7477-87.

[18] Kanapitsas A, Pissis P. Dielectric relaxation spectroscopy in crosslinked polyurethanes based on polymer polyols. European polymer journal 2000;36(6):1241-50.

[19] Petrovic ZS, Javni I, Divjakovic V. Structure and physical properties of segmented polyurethane elastomers containing chemical crosslinks in the hard segment. Journal of Polymer Science Part B Polymer Physics 1998;36(2):221-35.

[20] Valentová H, Nedbal J, Ilavskỳ M, Pissis P. Dsc, dielectric and dynamic mechanical behavior of two-and three-component ordered polyurethanes. Polymer 2005;46(12):4175-82.

[21] Pissis P, Kanapitsas A, Savelyev YV, Akhranovich E, Privalko E, Privalko V. Influence of chain extenders and chain end groups on properties of segmented polyurethanes. ii. dielectric study. Polymer 1998;39(15):3431-5.

[22] Jomaa M, Seveyrat L, Lebrun L, Masenelli-Varlot K, Cavaille J. Dielectric properties of segmented polyurethanes for electromechanical applications. Polymer 2015;63:214-21.

[23] Acierno D, Amendola E, Carfagna C, Concilio S, Iannelli P, Incarnato $\mathrm{L}$, et al. Synthesis and characterisation of a nematic homo-polyurethane. Polymer 2003;44(17):4949-58. 
[24] Alegria A, Goitiandia L, Colmenero J. Interpretation of the tsdc fractional polarization experiments on the $\alpha$-relaxation of polymers. Journal of Polymer Science Part B: Polymer Physics 2000;38(16):2105-13. 